\title{
Influence of Chemical Reaction on Heat and Mass Transfer Flow of a Micropolar Fluid over a Permeable Channel with Radiation and Heat Generation
}

\author{
Khilap Singh and Manoj Kumar \\ Department of Mathematics, Statistics and Computer Science, G. B. Pant University of Agriculture and Technology, \\ Pantnagar, Uttarakhand, India \\ Correspondence should be addressed to Khilap Singh; singh.khilap8@gmail.com
}

Received 30 June 2016; Accepted 9 November 2016

Academic Editor: Felix Sharipov

Copyright ( 92016 K. Singh and M. Kumar. This is an open access article distributed under the Creative Commons Attribution License, which permits unrestricted use, distribution, and reproduction in any medium, provided the original work is properly cited.

\begin{abstract}
The effects of chemical reaction on heat and mass transfer flow of a micropolar fluid in a permeable channel with heat generation and thermal radiation is studied. The Rosseland approximations are used to describe the radiative heat flux in the energy equation. The model contains nonlinear coupled partial differential equations which have been transformed into ordinary differential equation by using the similarity variables. The relevant nonlinear equations have been solved by Runge-Kutta-Fehlberg fourth fifth-order method with shooting technique. The physical significance of interesting parameters on the flow and heat transfer characteristics as well as the local skin friction coefficient, wall couple stress, and the heat transfer rate are thoroughly examined.
\end{abstract}

\section{Introduction}

The micropolar fluid theory is the one of the most important non-Newtonian fluid models described by Eringen [1]. This theory shows microrotation effects as well as microinertia and has many applications such as polymer fluids, liquid crystals, animal bloods, unusual lubricants, colloidal and suspension solutions, colloidal fluids, liquid crystals, and polymeric suspension. The extensive reviews of the micropolar fluid theory and its applications can be found in Eringen [2] and Lukaszewicz [3]. The unsteady mixed convection flow of a micropolar fluid from a vertical surface in the presence of viscous dissipation and the buoyancy force has been studied by El-Aziz [4]. Ashraf et al. [5] investigated the micropolar fluids flow through a porous channel. Sheikholeslami et al. [6] proposed the flow and heat transfer of micropolar fluid in a permeable channel. Prakash and Muthtamilselvan [7] investigated radiation effect on MHD micropolar fluid flow in porous vertical channel. Darvishi et al. [8] analyzed numerically the micropolar fluid flow from a porous channel. Sherief et al. [9] studied the motion along axis of a circular cylindrical pore in a micropolar fluid of a slip spherical particle. Mosayebidorcheh [10] analyzed the flow of micropolar fluid over a porous channel with changing walls. Recently, many authors [11-14] have studied the micropolar fluid flow for different fluid properties over different geometries. However, the effects of chemical reaction on micropolar fluid flow over a permeable channel in the presence of radiation and heat generation have not been considered in the above investigations.

Combined heat and mass transfer flows in the presence of chemical reaction have numerous applications in engineering and geophysics such as drying, geothermal reservoirs, dehydration at the surface of a water body, drying of porous solids, geothermal pool, thermal insulation, enhanced oil recovery, cooling of nuclear reactors, fibrous insulation, evaporation at the surface of a water body, pollution studies, cooling the polymer production and manufacturing of ceramics, energy transfer in a wet cooling tower, the flow in a desert, and oxidation and synthesis materials. Mohamed and Abo-Dahab [15] studied the effects of chemical reaction and thermal radiation on hydromagnetic free convection heat and mass transfer for a micropolar fluid bounded by a semi-infinite vertical porous plate in the presence of heat generation. In recent years, many researchers have studied and reported the effect of first-order chemical reaction [16-25]. 
Influence of thermal radiation on flow and heat transfer study has become more important industrially. The heat transfer and temperature profile of a micropolar fluid over different geometries can be affected significantly at high temperature. Bhattacharyya et al. [26] considered thermal radiation effect on micropolar fluid flow and heat transfer over a porous shrinking sheet. Hussain et al. [27] analyzed radiation effects on the thermal boundary layer flow of a micropolar fluid towards a permeable stretching sheet. Oahimire and Olajuwon [28] investigated the influence of Hall current and thermal radiation on heat and mass transfer of a chemically reacting MHD flow of a micropolar fluid through a porous medium. Mabood et al. [29] studied effects of nonuniform heat source/sink and Soret on MHD non-Darcian convective flow past a stretching sheet in a micropolar fluid with radiation.

The effect of heat generation on heat transfer is an important issue in view of various physical problems. Ziabakhsh et al. [30] analyzed the micropolar fluid flow with heat generation. Singh and Kumar [31] considered the melting effect in stagnation-point flow of micropolar fluid towards a stretching/shrinking surface. Bakr [32] investigated the effects of chemical reaction and heat source magnetoconvection and mass transfer flow of a micropolar fluid in a rotating frame of reference. The heat generation/absorption effects on MHD flow and heat transfer of micropolar fluid through a stretching surface have been proposed by Mahmoud and Waheed [33]. Abbasi et al. [34] examined the flow of Maxwell nanofluid in the presence of heat generation/absorption. Mliki et al. [35] investigated the influence of nanoparticle Brownian motion and heat generation/absorption over linear/sinusoidally heated cavity in the presence of magnetohydrodynamic natural convection. Sheikholeslami and Ganji [36] studied three-dimensional heat and mass transfer flow of nanofluid over a rotating system. Thermal radiation effects on mixed convection flow and heat transfer of a micropolar fluid through an unsteady stretching surface with heat generation/absorption are presented by Singh and Kumar [37].

Motivated by the above studies and applications, the present work explores effects of chemical reaction on heat and mass transfer flow of a micropolar fluid over a permeable channel in the presence of radiation and heat generation. The equations of continuity, momentum, angular momentum, energy, and concentration have been reduced to a system of nonlinear ordinary differential equations by similarity transforms which are solved by Runge-Kutta-Fehlberg method with shooting technique. It is expected that the results obtained from present paper will provide important information to the audience. To the best of our knowledge, such type of study is not investigated before in the scientific literature.

\section{Mathematical Formulation}

The heat and mass transfer flow of a micropolar fluid in a permeable channel with chemical reaction is considered in the present work. The thermal radiation and heat source are incorporated in the energy equation. The graphical model of

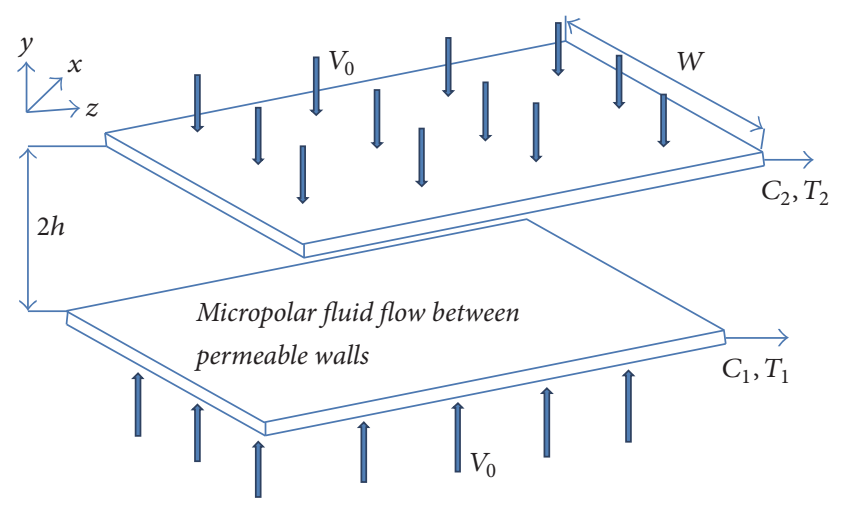

(a)

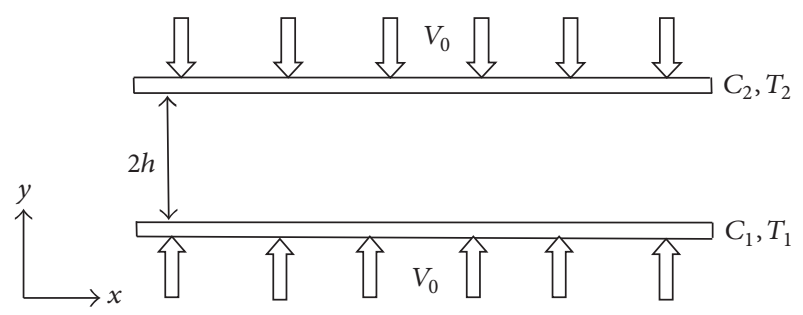

(b)

Figure 1: (a) Geometry of problem. (b) $x-y$ view at $z=W / 2$.

the problem has been given along with flow configuration and coordinate system in Figure 1. The assumptions of the problem in detail can be found in [6]. The governing equations of boundary layer are given in the following form:

$$
\begin{aligned}
\frac{\partial u}{\partial x}+\frac{\partial v}{\partial y}= & 0 \\
u \frac{\partial u}{\partial x}+v \frac{\partial u}{\partial y}= & -\frac{1}{\rho} \frac{\partial P}{\partial x}+\frac{(\mu+\kappa)}{\rho}\left(\frac{\partial^{2} u}{\partial x^{2}}+\frac{\partial^{2} u}{\partial y^{2}}\right) \\
& +\frac{\kappa}{\rho} \frac{\partial N}{\partial y}, \\
u \frac{\partial v}{\partial x}+v \frac{\partial v}{\partial y}= & -\frac{1}{\rho} \frac{\partial P}{\partial y}+\frac{(\mu+\kappa)}{\rho}\left(\frac{\partial^{2} v}{\partial x^{2}}+\frac{\partial^{2} v}{\partial y^{2}}\right) \\
& -\frac{\kappa}{\rho} \frac{\partial N}{\partial y}, \\
u \frac{\partial N}{\partial x}+v \frac{\partial N}{\partial y}= & \frac{\mu_{s}}{\rho j}\left(\frac{\partial^{2} N}{\partial x^{2}}+\frac{\partial^{2} N}{\partial y^{2}}\right) \\
& -\frac{\kappa}{\rho j}\left(2 N+\frac{\partial u}{\partial y}-\frac{\partial v}{\partial x}\right), \\
u \frac{\partial C}{\partial x}+v \frac{\partial C}{\partial y}= & D^{*} \frac{\partial^{2} C}{\partial y^{2}}-\gamma_{0}\left(C-C_{2}\right), \\
u \frac{\partial T}{\partial x}+v \frac{\partial^{2} T}{\partial y}= & \frac{\partial^{2}}{\rho C_{p}} \frac{\partial q_{r}}{\partial y^{2}}+\frac{\mu}{\rho C_{p}}\left(\frac{\partial u}{\partial y}\right)^{2} \\
& +\frac{Q_{0}}{\rho C_{p}}\left(T-T_{2}\right),
\end{aligned}
$$


where $u$ and $v$ indicate the velocity components in the $x$ and $y$ directions, respectively, $\rho$ is the fluid density, $\mu$ is the dynamic viscosity, $\kappa$ is the material parameter, $N$ is the angular or microrotation velocity, $P$ is the fluid pressure, $j$ is the microinertia density, $\mu_{s}=(\mu+\kappa / 2) j$ is the microrotation viscosity, $T$ is the fluid temperature, $C_{p}$ is the specific heat at constant pressure, $C$ is the fluid concentration, $k$ is the thermal conductivity, $q_{r}$ is the radiative heat flux, $Q_{0}$ is the heat generation coefficient, $D^{*}$ is the molecular diffusivity, and $\gamma_{0}$ is the chemical reaction rate coefficient.

Using Rosseland's approximation for radiation, we obtain

$$
q_{r}=-\left(\frac{4 \sigma}{3 k_{0}}\right) \frac{\partial T^{4}}{\partial y},
$$

where $\sigma$ is the Stefan-Boltzmann constant and $k_{0}$ is the absorption coefficient. We consider that the temperature variation within the flow is such that $T^{4}$ may be expanded in a Taylor's series. Expanding $T^{4}$ about $T_{\infty}$ and neglecting higher order terms, we get $T^{4}=4 T_{\infty}^{3} T-3 T_{\infty}^{4}$. Now (5) reduces to

$$
\begin{aligned}
u \frac{\partial T}{\partial x}+v \frac{\partial T}{\partial y}= & \frac{k}{\rho C_{p}} \frac{\partial^{2} T}{\partial y^{2}}+\frac{16 \sigma T_{\infty}^{3}}{3 k_{1} \rho C_{p}} \frac{\partial^{2} T}{\partial y^{2}} \\
& +\frac{\mu}{\rho C_{p}}\left(\frac{\partial u}{\partial y}\right)^{2}+\frac{Q_{0}}{\rho C_{p}}\left(T-T_{2}\right) .
\end{aligned}
$$

The appropriate boundary conditions for the flow are

$$
\begin{aligned}
& u=v=0, \\
& N=-n \frac{\partial u}{\partial y}, \\
& T=T_{1}, \\
& C=C_{1}, \\
& u=\frac{v_{0} x}{h}, \\
& v=0, \\
& N=\frac{v_{0} x}{h^{2}}, \\
& T=T_{2}, \\
& C=C_{2},
\end{aligned}
$$

$$
\text { at } y=-h \text {, }
$$

where boundary parameters $n(0 \leq n \leq 1)$ indicate the degree to which the microelements are free to rotate near the channel walls. The case when $n=0$ is called strong concentration of microelements, which implies $N=0$ near the wall surface. This represents concentrated particle flow where the microelements close to the wall surface are unable to rotate. In the case when $n=1 / 2$, this indicates vanishing of the antisymmetric part of the stress tensor and denoted weak concentration of microelement and $n=1$ is used for the modeling of turbulent boundary layer flow. In this paper, the authors considered $n=1 / 2$ for which the governing equations can be reduced to the classical problem of steady boundary layer flow of a viscous incompressible fluid near the channel wall.

Equations (2), (3), (4), (6), and (8) can be transformed into a set of nonlinear ordinary differential equations by using the following similarity transformations:

$$
\begin{aligned}
\eta & =\frac{y}{h}, \\
\psi & =-v_{0} x f(\eta), \\
N & =\frac{v_{0} x}{h^{2}} g(\eta), \\
\theta(\eta) & =\frac{T-T_{2}}{T_{1}-T_{2}}, \\
\phi(\eta) & =\frac{C-C_{2}}{C_{1}-C_{2}},
\end{aligned}
$$

where $T_{2}=T_{1}-A x$ and $C_{2}=C_{1}-B x$, with $A$ and $B$ as constants. The stream function $\psi$ is defined as

$$
\begin{aligned}
& u=\frac{\partial \psi}{\partial y}, \\
& v=-\frac{\partial \psi}{\partial x} .
\end{aligned}
$$

The coupled system of transformed nonlinear ordinary differential equations is

$$
\begin{aligned}
\left(1+N_{1}\right) f^{\mathrm{IV}}-N_{1} g-\operatorname{Re}\left(f f^{\prime \prime \prime}-f^{\prime} f^{\prime \prime}\right) & =0, \\
N_{2} g^{\prime \prime}+N_{1}\left(f^{\prime \prime}-2 g\right)-N_{3} \operatorname{Re}\left(f g^{\prime}-f^{\prime} g\right) & =0, \\
(1+R) \theta^{\prime \prime}+\mathrm{Pe}_{h}\left[\mathrm{Ec} f^{\prime \prime^{2}}+f^{\prime} \theta-f \theta^{\prime}+H \theta\right] & =0, \\
\phi^{\prime \prime}+\mathrm{Pe}_{m}\left(f^{\prime} \phi-f \phi^{\prime}-\gamma \phi\right) & =0 .
\end{aligned}
$$

Boundary conditions in nondimensional form are

$$
\begin{aligned}
f(-1) & =f^{\prime}(-1)=g(-1)=0, \\
\theta(-1) & =\phi(-1)=1, \\
f(1) & =\theta(1)=\phi(1)=0, \\
f^{\prime}(1) & =-1, \\
g(1) & =1,
\end{aligned}
$$

where $N_{1}=\kappa / \mu$ is the coupling number, $N_{2}=v_{s} / \mu h^{2}$ is the spin-gradient viscosity parameter, $N_{3}=j / h^{2}$ is the micropolar material constant, $\mathrm{Ec}=v_{0}^{2} x v / h^{3} c_{p} A$ is the local Eckert number, $H=\theta_{0} h / \rho C_{p} v_{0}$ is the heat generation parameter, $\mathrm{Pe}_{h}=\operatorname{PrRe}$ and $\mathrm{Pe}_{m}=\mathrm{ScRe}$ are the Peclet numbers for the diffusion of heat and the diffusion of mass, $\operatorname{Re}=\left(v_{0} / v\right) h$ 
is the Reynolds number, $R=3 k_{0} k / 16 \sigma T_{\infty}^{3}$ is the thermal radiation parameter, $\mathrm{Gr}=g \beta_{T} A h^{4} / v^{2}$ is the Grashof number, $\operatorname{Pr}=v \rho c_{p} / k$ is the Prandtl number, Sc $=v / D^{*}$ is the Schmidt number, and $\gamma=\gamma_{0} h / v_{0}$ is the chemical reaction parameter.

The other parameters of physical interest are the local Nusselt $\mathrm{Nu}_{x}$ and Sherwood $\mathrm{Sh}_{x}$ numbers, which are defined as follows:

$$
\begin{aligned}
\mathrm{Nu}_{x} & =\frac{x q_{w}}{k\left(T_{1}-T_{2}\right)}, \\
\mathrm{Sh}_{x} & =\frac{x m_{w}}{D^{*}\left(C_{1}-C_{2}\right)},
\end{aligned}
$$

where $q_{w}$ and $m_{w}$ are the local heat flux and mass flux, respectively, which are defined as

$$
\begin{aligned}
& q_{w}=-k\left(\frac{\partial T}{\partial y}\right)_{y=-h}, \\
& m_{w}=D^{*}\left(\frac{\partial C}{\partial y}\right)_{y=-h} .
\end{aligned}
$$

Now using (10) and (15) in (14), we get

$$
\begin{aligned}
& \mathrm{Nu}_{x}=-\theta^{\prime}(-1), \\
& \mathrm{Sh}_{x}=-\phi^{\prime}(-1) .
\end{aligned}
$$

\section{Method of Solution}

In this present paper, Runge-Kutta-Fehlberg fourth fifthorder method has been employed to solve the system of nonlinear ordinary differential equations (12) with the boundary conditions given by (13) for different values of governing parameters. The RKF 45 method has a procedure to determine if the appropriate step size $h$ is being used. The formula of fifth-order Runge-Kutta-Fehlberg method can be defined as follows:

$$
\begin{aligned}
& z_{n+1}=z_{n}+\left(\frac{16}{135} k_{0}+\frac{6656}{12,825} k_{2}+\frac{28,561}{56,430} k_{3}-\frac{9}{50} k_{4}\right. \\
& \left.+\frac{2}{55} k_{5}\right) h,
\end{aligned}
$$

where the coefficients $k_{0}$ to $k_{5}$ are given by

$$
\begin{aligned}
k_{0} & =f\left(x_{n}, y_{n}\right), \\
k_{1} & =f\left(x_{n}+\frac{1}{4} h, y_{n}+\frac{1}{4} h k_{0}\right), \\
k_{2} & =f\left(x_{n}+\frac{3}{8} h, y_{n}+\left(\frac{3}{32} k_{0}+\frac{9}{32} k_{1}\right) h\right),
\end{aligned}
$$

$$
\begin{aligned}
k_{3} & =f\left(x_{n}+\frac{12}{13} h, y_{n}\right. \\
& \left.+\left(\frac{1932}{2197} k_{0}-\frac{7200}{2197} k_{1}+\frac{7296}{2197} k_{2}\right) h\right), \\
k_{4} & =f\left(x_{n}+h, y_{n}\right. \\
& \left.+\left(\frac{439}{216} k_{0}-8 k_{1}+\frac{3680}{513} k_{2}-\frac{845}{4104} k_{3}\right) h\right), \\
k_{5} & =f\left(x_{n}+\frac{1}{2} h, y_{n}\right. \\
& \left.+\left(-\frac{8}{27} k_{0}+2 k_{1}-\frac{3544}{2565} k_{2}+\frac{1859}{4104} k_{3}-\frac{11}{40} k_{4}\right) h\right) .
\end{aligned}
$$

The computation of the error can be achieved by subtracting the fifth-order from the fourth-order method:

$$
y_{n+1}=y_{n}+\left(\frac{25}{216} k_{0}+\frac{1408}{2565} k_{2}+\frac{2197}{4101} k_{3}-\frac{1}{5} k_{4}\right) h .
$$

If the error goes beyond a specified antechamber, the results can be recalculated using a smaller step size. The approach to computing the new step size is shown as follows:

$$
h_{\text {new }}=h_{\text {old }}\left(\frac{\varepsilon h_{\text {old }}}{2\left|z_{n+1}-y_{n+1}\right|}\right)^{1 / 4} \text {. }
$$

The numerical computations were carried out with $\Delta \eta=0.01$. The variation of the dimensionless velocity, microrotation, temperature, and concentration is ensured to be less than $10^{-6}$ between any two successive iterations for the convergence criterion.

\section{Results and Discussion}

In order to study, the effects of various governing physical parameters on the flow, heat, and mass transfer numerical computations are carried out for $0.1 \leq N_{1}=N_{2}=N_{3} \leq 1$, $0 \leq H \leq 10,-2 \leq \operatorname{Re} \leq 5,0 \leq R \leq 4,0 \leq \mathrm{Pe}_{h} \leq 2,0 \leq$ $\mathrm{Pe}_{m} \leq 2$, and $0 \leq \gamma \leq 10$ while the Eckert number Ec $=0.01$ is fixed. A critical analysis with previously published work is done in Table 1 and these results are found to be in very good agreement.

The effects of the Reynolds number Re on velocity $f^{\prime}(\eta)$ and microrotation $g(\eta)$ are shown in Figures 2 and 3. It is seen from Figure 2 that velocity profile $f^{\prime}(\eta)$ decreases near the lower channel wall, while it increases near the upper channel wall when the Reynolds number Re increases. It is clear from Figure 3 that an increase in the magnitude of Re leads to decrease in microrotation profile $g(\eta)$. 
TABLE 1: Comparison of values of $\phi(\eta)$ for various values of $\eta, \mathrm{Pe}_{m}$, and $\operatorname{Re}$ when $\mathrm{Pe}_{h}=0.2, N_{1}=N_{2}=N_{3}=0.1, N_{1}=N_{2}=N_{3}=0.1$, and $\mathrm{Ec}=R=\gamma=0$.

\begin{tabular}{lcccc}
\hline & \multicolumn{2}{c}{ Sheikholeslami et al. [6] } & \multicolumn{2}{c}{ Present results } \\
& $\mathrm{Pe}_{m}=0.2, \mathrm{Re}=0.5$ & $\mathrm{Pe}_{m}=0.5, \mathrm{Re}=1$ & $\mathrm{Pe}_{m}=0.2, \mathrm{Re}=0.5$ & 1 \\
-1.0 & 1 & 1 & 0.814346 & 0.835992 \\
-0.6 & 0.814341 & 0.835986 & 0.621165 & 0.653501 \\
-0.2 & 0.621160 & 0.653497 & 0.521114 & 0.553590 \\
0.0 & 0.521109 & 0.553582 & 0.419039 & 0.448499 \\
0.2 & 0.419033 & 0.448495 & 0.210684 & 0.227358 \\
0.6 & 0.210678 & 0.227352 & 0 & 0 \\
1.0 & 0 & 0 & 0 & 0.5 \\
\hline
\end{tabular}

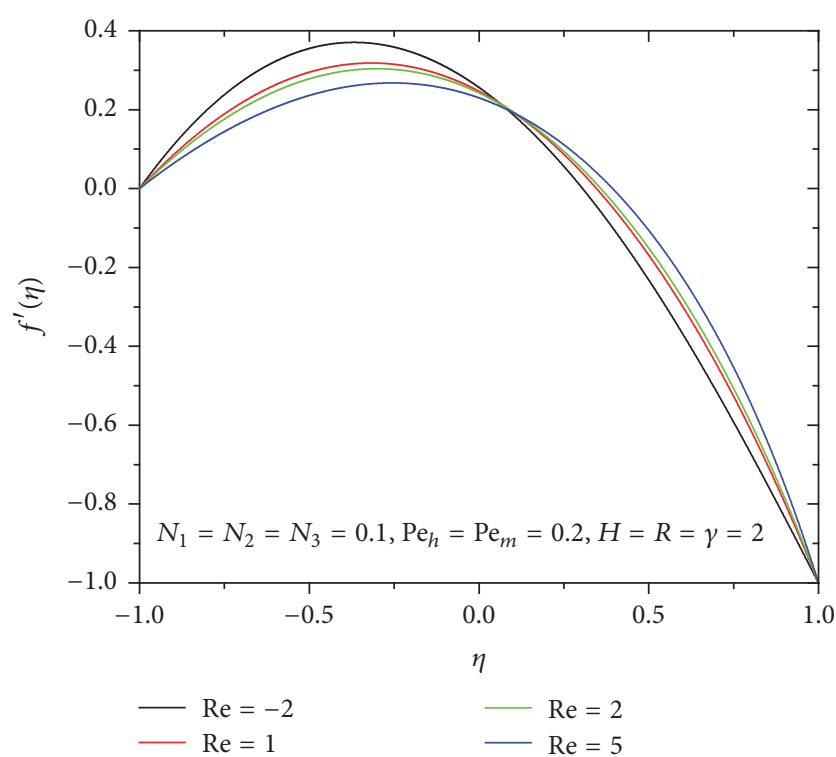

FIGURE 2: Velocity profile $f^{\prime}(\eta)$ for various values of Re.

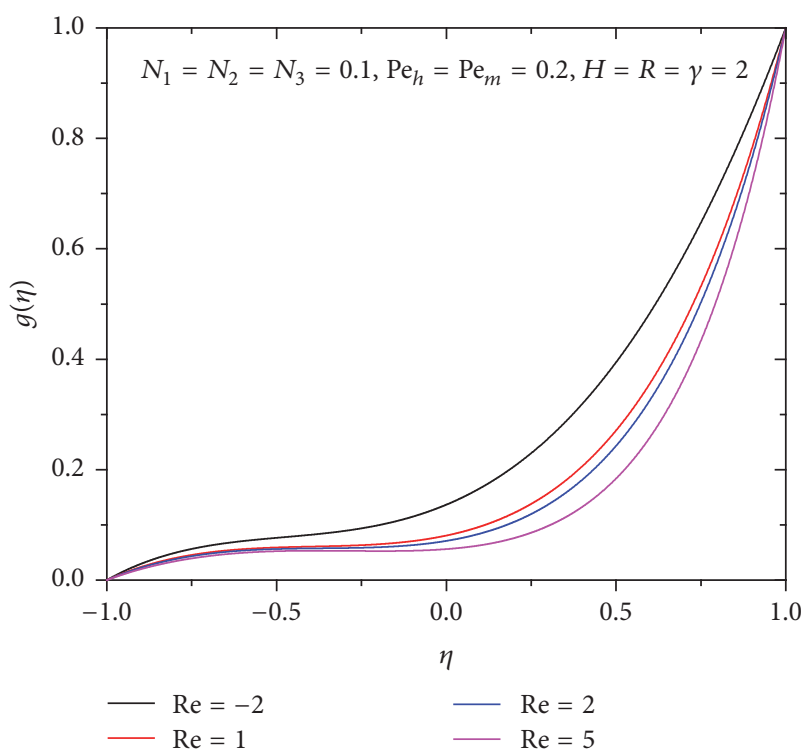

FIGURE 3: Microrotation profile $g(\eta)$ for various values of Re.

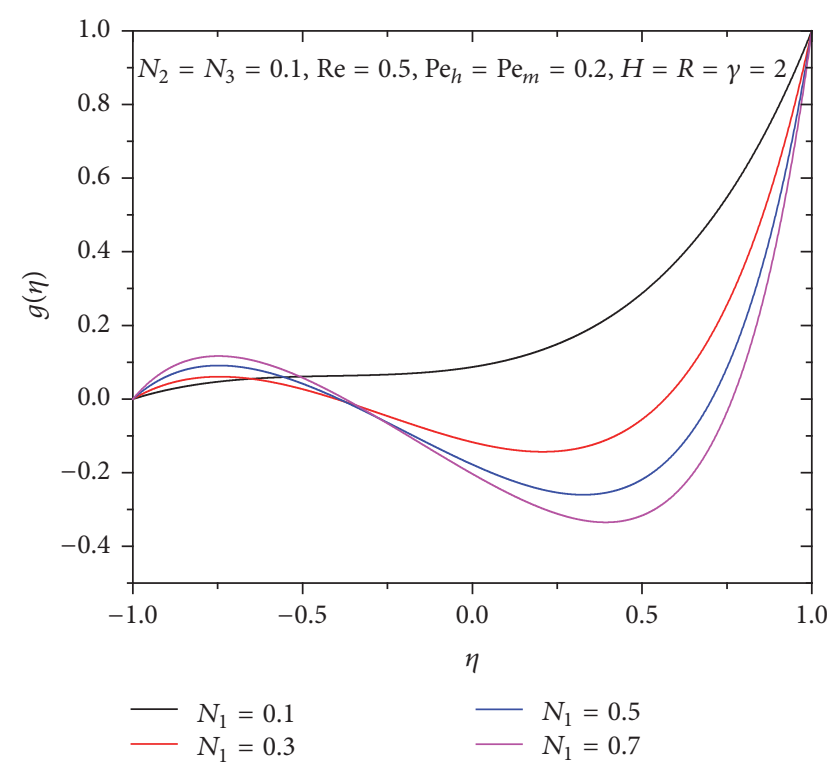

FIGURE 4: Microrotation profile $g(\eta)$ for various values of $N_{1}$.

The microrotation profile $g(\eta)$ for the coupling number $N_{1}$, the spin-gradient viscosity parameter $N_{2}$, and the micropolar material constant $N_{3}$ are displayed in Figures 46. It is noticed from Figure 4 that the microrotation profile increases with $N_{1}$ at lower channel wall for $0 \leq \eta<-0.65$, but reverse trend is found for the case $\eta>-0.65$. It is depicted from Figure 5 that the value of microrotation profile $g(\eta)$ increases as the value of $N_{2}$ increases. It is evident from Figure 6 that the values of microrotation are lower for higher values of $\mathrm{N}_{3}$.

Figures 7-9 show the temperature distribution $\theta(\eta)$ with collective variation in heat source parameter $H$, thermal radiation parameter $R$, and Peclet number $\mathrm{Pe}_{h}$. Figure 7 exhibits that temperature $\theta(\eta)$ considerably increases with an increase in $H$. Figure 8 indicates that an increase in $R$ induces a decrease in temperature $\theta(\eta)$. It is noticed from Figure 9 that a rise in $\mathrm{Pe}_{h}$ causes rapid increase in temperature.

The effects of the Peclet number $\mathrm{Pe}_{m}$ and chemical reaction parameter $\gamma$ on the concentration profile $\phi(\eta)$ are shown in Figures 10 and 11. Figures 10 and 11 indicate that 


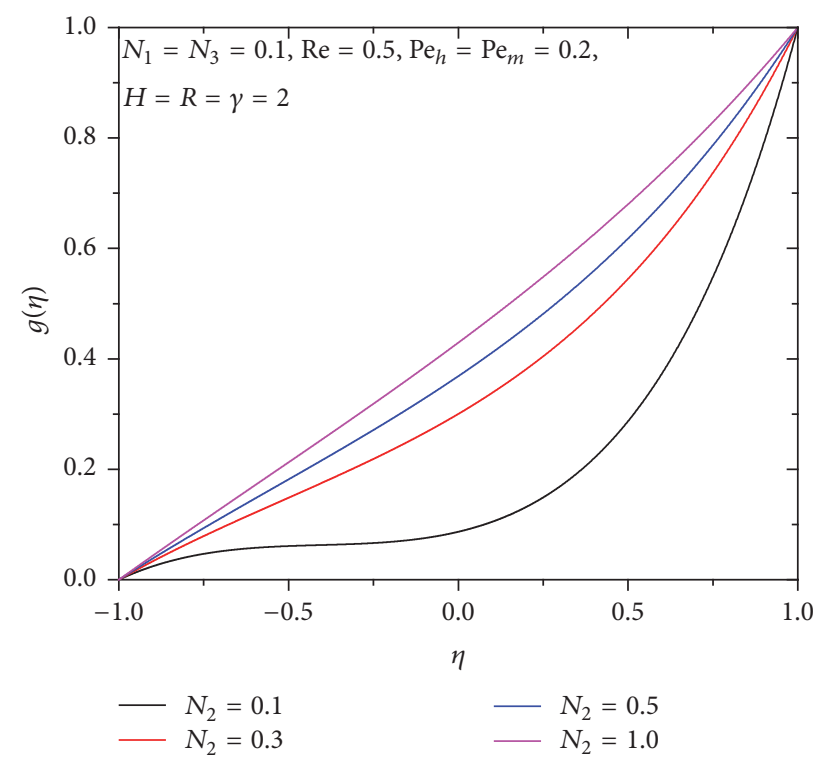

Figure 5: Microrotation profile $g(\eta)$ for various values of $N_{2}$.

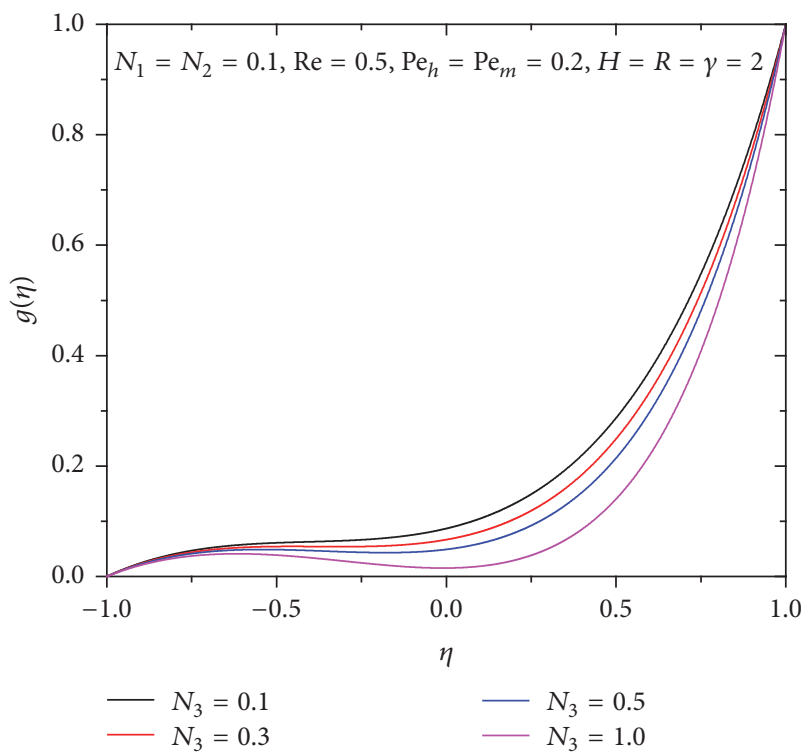

Figure 6: Microrotation profile $g(\eta)$ for various values of $N_{3}$.

concentration decreases with increasing values of $\mathrm{Pe}_{m}$ and $\gamma$.

The variation of the Nusselt number (dimensionless heat transfer rate at the surface) is displayed for different parameters in Figures 12-14. Figure 12 depicts the behavior of the Nusselt's number against Reynolds number Re with various values of heat source parameter $H$. It is clear that, with the increasing of $H$, the heat transfer rate decreases. Figure 13 enlightens the variation of the heat transfer rates with Reynolds number Re for various values of thermal radiation parameter $R$. It is evident from Figure 13 that Nusselt number monotonically increases with a rise in $R$ values. Figure 14 depicts the variation of Nusselt number as function of Reynolds number Re and Peclet number $\mathrm{Pe}_{h}$. It

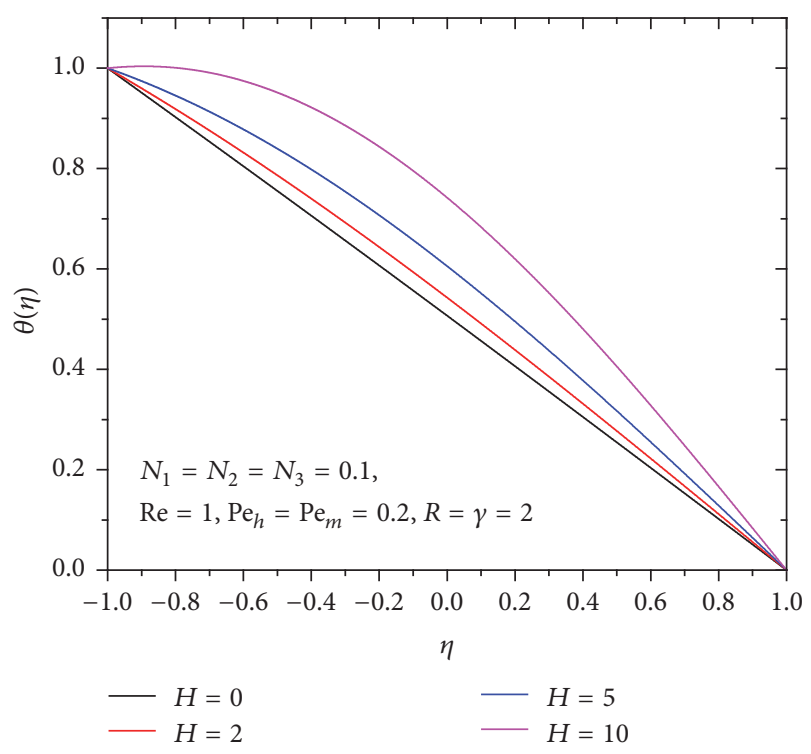

Figure 7: Temperature profile $\theta(\eta)$ for various values of $H$.

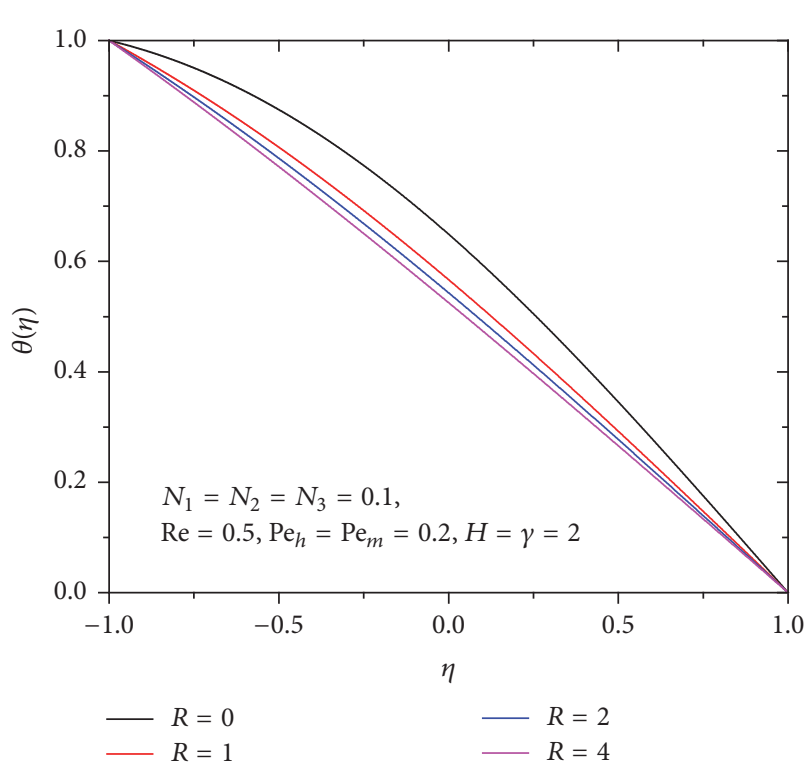

Figure 8: Temperature profile $\theta(\eta)$ for various values of $R$.

is noted that heat transfer rate decreases with a rise in $\mathrm{Pe}_{h}$ values. Also, a negligible change in heat transfer rate with increasing values of Reynolds number Re is seen from Figures 12-14.

Figures 15 and 16 displayed the variation of the Sherwood number or mass transfer rate for different parameters. Figures 15 and 16 depict the behavior of the mass transfer rate against Reynolds number Re with different values of Peclet number $\mathrm{Pe}_{m}$ and chemical reaction parameter $\gamma$. The mass transfer rate rises with increasing values of the Peclet number $\mathrm{Pe}_{m}$ and chemical reaction parameter $\gamma$. It is also clear from these figures that there is no change in mass transfer rate with rise in Reynolds number Re values. 


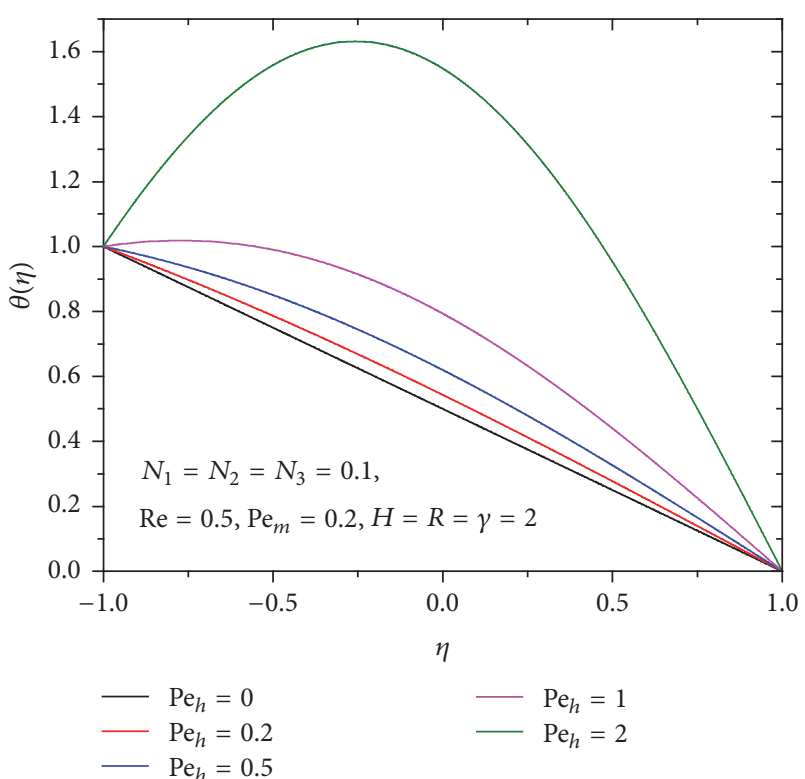

Figure 9: Temperature profile $\theta(\eta)$ for various values of $\mathrm{Pe}_{h}$.

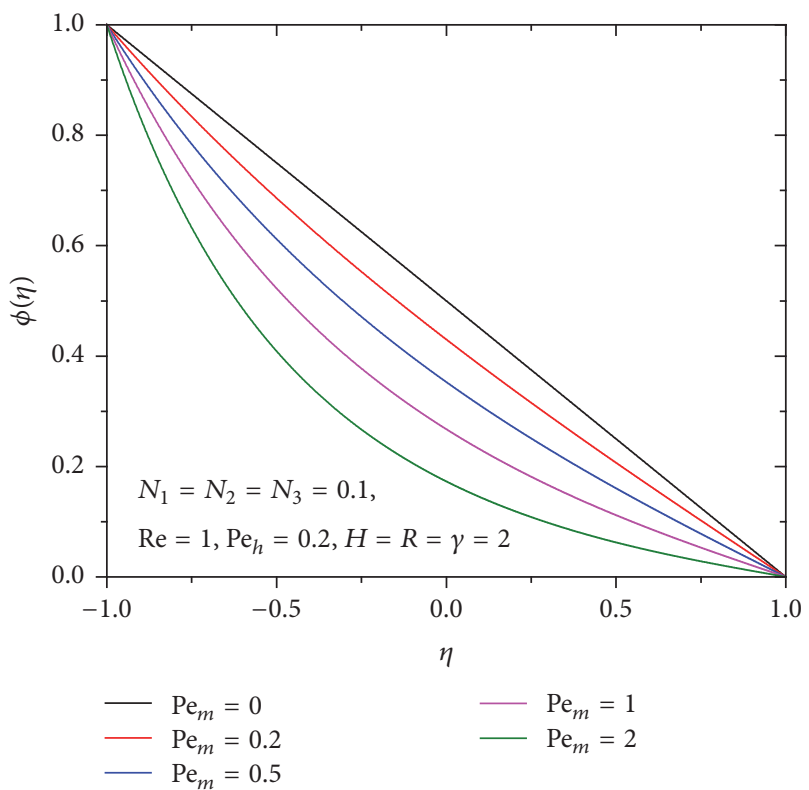

Figure 10: Concentration profile $\phi(\eta)$ for various values of $\mathrm{Pe}_{m}$.

\section{Conclusions}

The present paper deals with numerical analysis of chemical reaction effects on heat and mass transfer flow of a micropolar fluid over a permeable channel in the presence of radiation and heat generation. The system of nonlinear partial differential equations was converted to a system of ordinary differential equations and then is solved numerically using the Runge-Kutta-Fehlberg method along with shooting method. From the above discussion, the important results are summarized as follows:

(i) Velocity and Reynolds number are inversely proportional to each other at lower channel wall, while

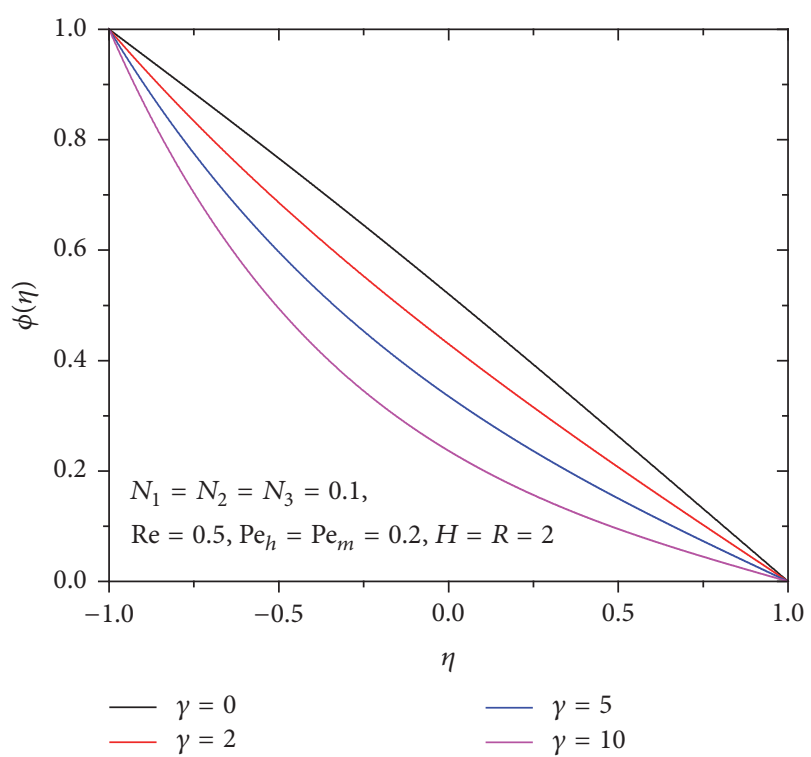

FIGURE 11: Concentration profile $\phi(\eta)$ for various values of $\gamma$.

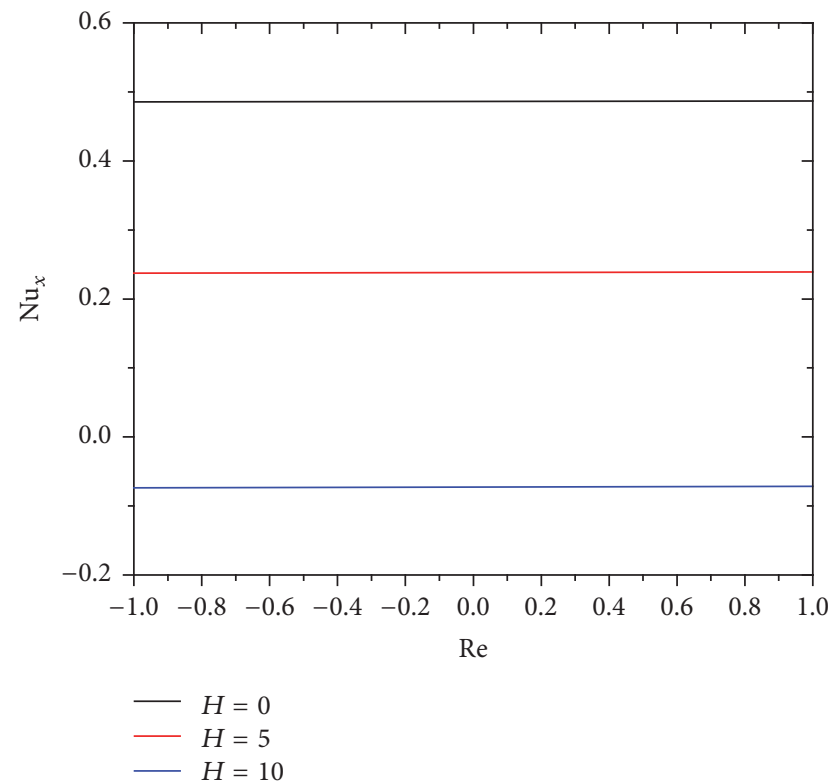

FIGURE 12: Variation of Nusselt's number with $H$ and Re.

velocity and Reynolds number are proportional to each other at upper channel wall.

(ii) Microrotation decreases with increase in the value of coupling number, micropolar material constant, and Reynolds number, but it increases with increase in the value of spin-gradient viscosity parameter.

(iii) Temperature increases with heat generation parameter and $\mathrm{Pe}_{h}$ and $\mathrm{Pe}_{m}$ are the Peclet numbers for the diffusion of heat and the diffusion of mass, and temperature decreases with thermal radiation parameter.

(iv) Concentration is inversely proportional to the Peclet number for the diffusion of mass and chemical reaction parameter. 


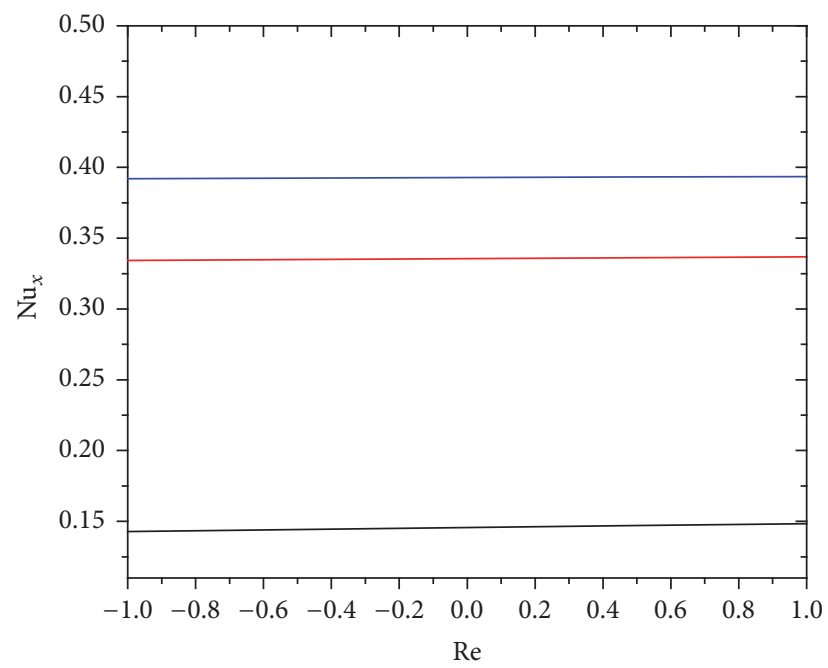

$$
\begin{aligned}
R & =0 \\
R & =2 \\
-R & =4
\end{aligned}
$$

Figure 13: Variation of Nusselt's number with $R$ and Re.

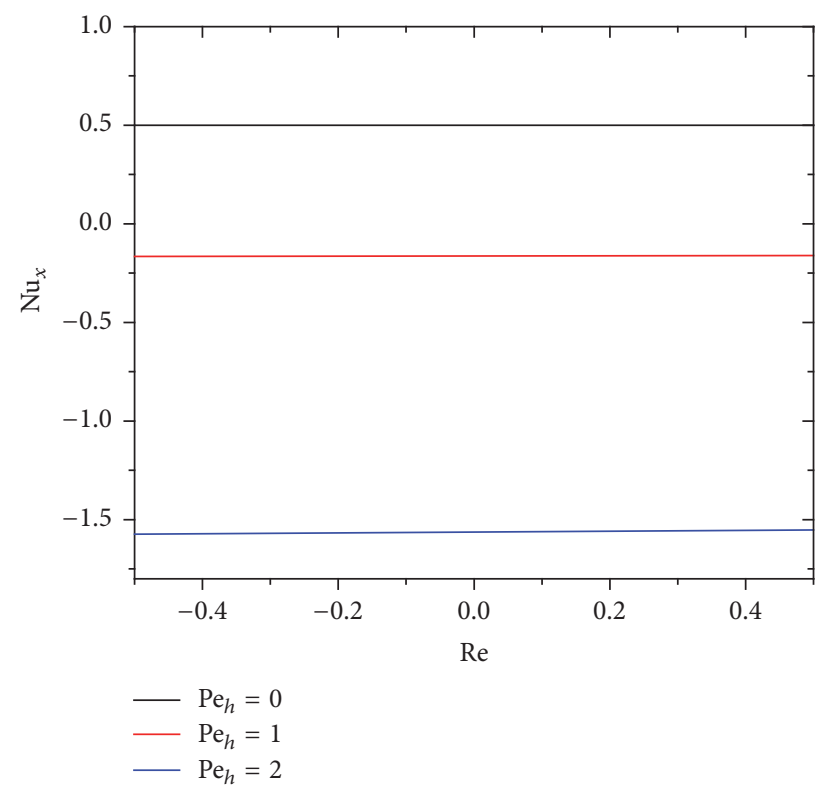

FIgURE 14: Variation of Nusselt's number with $\mathrm{Pe}_{h}$ and Re.

(v) The rate of heat transfer increases with thermal radiation parameter and rate of heat transfer decreases with Peclet number $\mathrm{Pe}_{h}$ and heat generation parameter.

(vi) The rate of mass transfer rises with increase Peclet number $\mathrm{Pe}_{m}$ and chemical reaction parameter.

\section{Nomenclature}

$C:$ Species concentration

$D^{*}$ : Molecular diffusivity

$f$ : Dimensionless stream function

$g:$ Dimensionless microrotation

$h$ : Width of the channel

j: Microinertia density

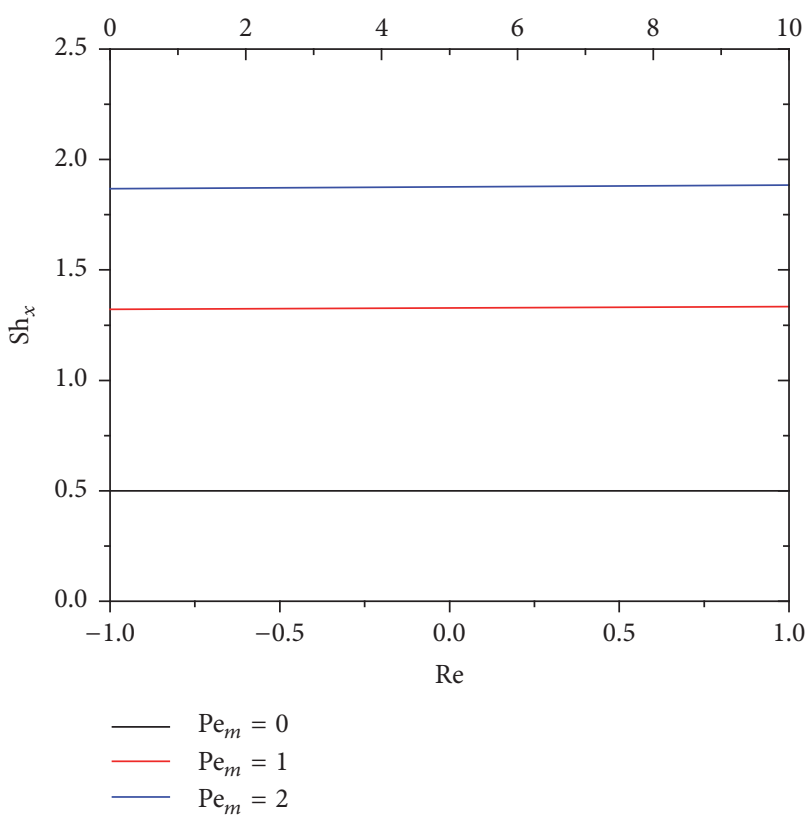

Figure 15: Variation of Sherwood number with $\mathrm{Pe}_{m}$ and Re.

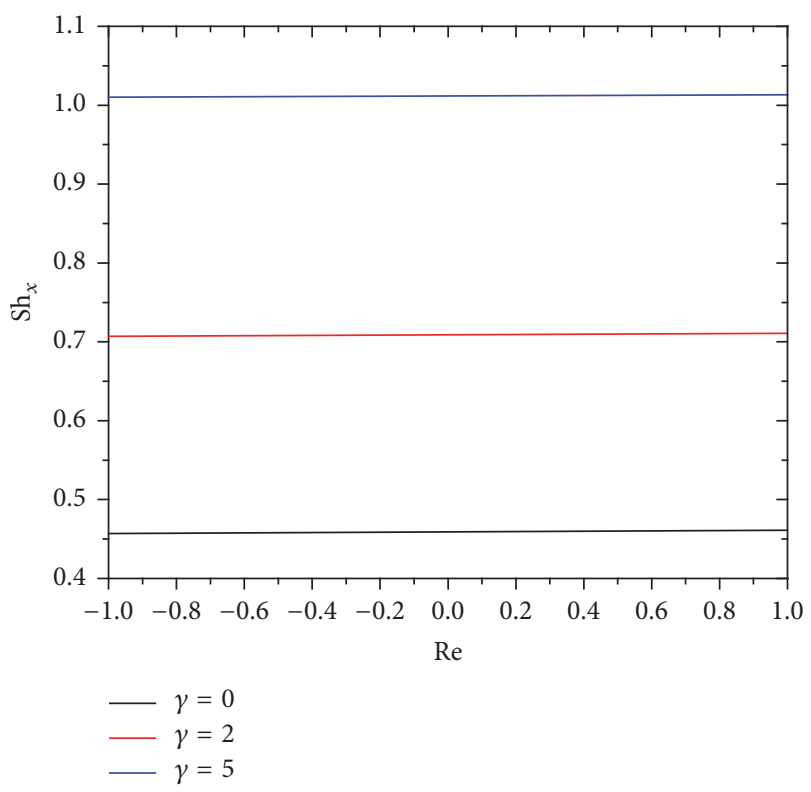

FIGURE 16: Variation of Sherwood number with $\gamma$ and Re.

N: Microrotation/angular velocity

$N_{1,2,3}$ : Dimensionless parameter

Nu: Nusselt number

Sh: Sherwood number

Sc: Schmidt number

p: $\quad$ Pressure

Pt: Prandtl number

Pe: Peclet number

q: $\quad$ Mass transfer parameter

$q_{r}: \quad$ Radiative heat flux

Re: Reynold number

T: $\quad$ Fluid temperature

$s$ : Microrotation boundary condition 
$(u, v)$ : Cartesian velocity components

$(x, y)$ : Cartesian coordinate components parallel and normal to cannel axis, respectively.

\section{Greek Symbols}

$\eta$ : Similarity variable

$\theta$ : Dimensionless temperature

$\mu$ : Dynamic viscosity

$\kappa:$ Material parameter

$\rho$ : Fluid density

$\mu_{s}:$ Microrotation/spin-gradient viscosity

$\psi$ : Stream function.

\section{Competing Interests}

The authors declare that there is no conflict of interests regarding the publication of this paper.

\section{Acknowledgments}

The first author gratefully acknowledges the financial support of UGC, India, under F. 17-97/2008 (SA-I) for pursuing this work.

\section{References}

[1] A. C. Eringen, "Theory of micropolar fluid," Jour of Mathematics and Mechanics, vol. 16, pp. 1-18, 1966.

[2] A. C. Eringen, "Theory of thermomicrofluids," Journal of Mathematical Analysis and Applications, vol. 38, no. 2, pp. 480496, 1972.

[3] G. Lukaszewicz, Micropolar Fluids: Theory and Application, Birkhäuser, Basel, Switzerland, 1999.

[4] M. A. El-Aziz, "Mixed convection flow of a micropolar fluid from an unsteady stretching surface with viscous dissipation," Journal of the Egyptian Mathematical Society, vol. 21, no. 3, pp. 385-394, 2013.

[5] M. Ashraf, M. A. Kamal, and K. S. Syed, "Numerical study of asymmetric laminar flow of micropolar fluids in a porous channel," Computers \& Fluids, vol. 38, no. 10, pp. 1895-1902, 2009.

[6] M. Sheikholeslami, M. Hatami, and D. D. Ganji, "Micropolar fluid flow and heat transfer in a permeable channel using analytical method," Journal of Molecular Liquids, vol. 194, pp. 30-36, 2014.

[7] D. Prakash and M. Muthtamilselvan, "Effect of radiation on transient MHD flow of micropolar fluid between porous vertical channel with boundary conditions of the third kind," Ain Shams Engineering Journal, vol. 5, no. 4, pp. 1277-1286, 2014.

[8] M. T. Darvishi, F. Khani, F. G. Awad, A. A. Khidir, and P. Sibanda, "Numerical investigation of the flow of a micropolar fluid through a porous channel with expanding or contracting walls," Propulsion and Power Research, vol. 3, no. 3, pp. 133-142, 2014.

[9] H. H. Sherief, M. S. Faltas, E. A. Ashmawy, and M. G. Nashwan, "Slow motion of a slip spherical particle along the axis of a circular cylindrical pore in a micropolar fluid," Journal of Molecular Liquids, vol. 200, pp. 273-282, 2014.
[10] S. Mosayebidorcheh, "Analytical investigation of the micropolar flow through a porous channel with changing walls," Journal of Molecular Liquids, vol. 196, pp. 113-119, 2014.

[11] M. Fakour, A. Vahabzadeh, D. D. Ganji, and M. Hatami, "Analytical study of micropolar fluid flow and heat transfer in a channel with permeable walls," Journal of Molecular Liquids, vol. 204, pp. 198-204, 2015.

[12] A. Tetbirt, M. N. Bouaziz, and M. T. Abbes, "Numerical study of magnetic effect on the velocity distribution field in a macro/micro-scale of a micropolar and viscous fluid in vertical channel," Journal of Molecular Liquids, vol. 216, pp. 103-110, 2016.

[13] M. Ramzan, M. Farooq, T. Hayat, and J. D. Chung, "Radiative and Joule heating effects in the MHD flow of a micropolar fluid with partial slip and convective boundary condition," Journal of Molecular Liquids, vol. 221, pp. 394-400, 2016.

[14] N. S. Gibanov, M. A. Sheremet, and I. Pop, "Natural convection of micropolar fluid in a wavy differentially heated cavity," Journal of Molecular Liquids, vol. 221, pp. 518-525, 2016.

[15] R. A. Mohamed and S. M. Abo-Dahab, "Influence of chemical reaction and thermal radiation on the heat and mass transfer in MHD micropolar flow over a vertical moving porous plate in a porous medium with heat generation," International Journal of Thermal Sciences, vol. 48, no. 9, pp. 1800-1813, 2009.

[16] E. Magyari and A. J. Chamkha, "Combined effect of heat generation or absorption and first-order chemical reaction on micropolar fluid flows over a uniformly stretched permeable surface: the full analytical solution," International Journal of Thermal Sciences, vol. 49, no. 9, pp. 1821-1828, 2010.

[17] K. Das, "Effect of chemical reaction and thermal radiation on heat and mass transfer flow of MHD micropolar fluid in a rotating frame of reference," International Journal of Heat and Mass Transfer, vol. 54, no. 15-16, pp. 3505-3513, 2011.

[18] A. A. Bakr, "Effects of chemical reaction on MHD free convection and mass transfer flow of a micropolar fluid with oscillatory plate velocity and constant heat source in a rotating frame of reference," Communications in Nonlinear Science and Numerical Simulation, vol. 16, no. 2, pp. 698-710, 2011.

[19] K. Das, "Influence of thermophoresis and chemical reaction on MHD micropolar fluid flow with variable fluid properties," International Journal of Heat and Mass Transfer, vol. 55, no. 2324, pp. 7166-7174, 2012.

[20] A. M. Rashad, S. Abbasbandy, and A. J. Chamkha, "Mixed convection flow of a micropolar fluid over a continuously moving vertical surface immersed in a thermally and solutally stratified medium with chemical reaction," Journal of the Taiwan Institute of Chemical Engineers, vol. 45, no. 5, pp. 2163-2169, 2014.

[21] F. Mabood, W. A. Khan, and A. I. M. Ismail, "MHD stagnation point flow and heat transfer impinging on stretching sheet with chemical reaction and transpiration," Chemical Engineering Journal, vol. 273, pp. 430-437, 2015.

[22] K. Singh and M. Kumar, "The effect of chemical reaction and double stratification on MHD free convection in a micropolar fluid with heat generation and Ohmic heating," Jordan Journal of Mechanical and Industrial Engineering, vol. 9, no. 4, pp. 279288, 2015.

[23] S. Srinivas, A. Gupta, S. Gulati, and A. S. Reddy, "Flow and mass transfer effects on viscous fluid in a porous channel with moving/stationary walls in presence of chemical reaction," International Communications in Heat and Mass Transfer, vol. 48, pp. 34-39, 2013. 
[24] J. C. Umavathi, M. A. Sheremet, and S. Mohiuddin, "Combined effect of variable viscosity and thermal conductivity on mixed convection flow of a viscous fluid in a vertical channel in the presence of first order chemical reaction," European Journal of Mechanics-B/Fluids, vol. 58, pp. 98-108, 2016.

[25] J. C. Misra and S. D. Adhikary, "MHD oscillatory channel flow, heat and mass transfer in a physiological fluid in presence of chemical reaction," Alexandria Engineering Journal, vol. 55, no. 1, pp. 287-297, 2016.

[26] K. Bhattacharyya, S. Mukhopadhyay, G. C. Layek, and I. Pop, "Effects of thermal radiation on micropolar fluid flow and heat transfer over a porous shrinking sheet," International Journal of Heat and Mass Transfer, vol. 55, no. 11-12, pp. 2945-2952, 2012.

[27] M. Hussain, M. Ashraf, S. Nadeem, and M. Khan, "Radiation effects on the thermal boundary layer flow of a micropolar fluid towards a permeable stretching sheet," Journal of the Franklin Institute, vol. 350, no. 1, pp. 194-210, 2013.

[28] J. I. Oahimire and B. I. Olajuwon, "Effect of Hall current and thermal radiation on heat and mass transfer of a chemically reacting MHD flow of a micropolar fluid through a porous medium," Journal of King Saud University-Engineering Sciences, vol. 26, no. 2, pp. 112-121, 2014.

[29] F. Mabood, S. M. Ibrahim, M. M. Rashidi, M. S. Shadloo, and G. Lorenzini, "Non-uniform heat source/sink and Soret effects on MHD non-Darcian convective flow past a stretching sheet in a micropolar fluid with radiation," International Journal of Heat and Mass Transfer, vol. 93, pp. 674-682, 2016.

[30] Z. Ziabakhsh, G. Domairry, and H. Bararnia, "Analytical solution of non-Newtonian micropolar fluid flow with uniform suction/blowing and heat generation," Journal of the Taiwan Institute of Chemical Engineers, vol. 40, no. 4, pp. 443-451, 2009.

[31] K. Singh and M. Kumar, "Melting heat transfer in boundary layer stagnation point flow of MHD micro-polar fluid towards a stretching/shrinking surface," Jordan Journal of Mechanical and Industrial Engineering, vol. 8, no. 6, pp. 403-408, 2014.

[32] A. A. Bakr, "Effects of chemical reaction on MHD free convection and mass transfer flow of a micropolar fluid with oscillatory plate velocity and constant heat source in a rotating frame of reference," Communications in Nonlinear Science and Numerical Simulation, vol. 16, no. 2, pp. 698-710, 2011.

[33] M. A. A. Mahmoud and S. E. Waheed, "MHD flow and heat transfer of a micropolar fluid over a stretching surface with heat generation (absorption) and slip velocity," Journal of the Egyptian Mathematical Society, vol. 20, no. 1, pp. 20-27, 2012.

[34] F. M. Abbasi, S. A. Shehzad, T. Hayat, and B. Ahmad, "Doubly stratified mixed convection flow of Maxwell nanofluid with heat generation/absorption," Journal of Magnetism and Magnetic Materials, vol. 404, pp. 159-165, 2016.

[35] B. Mliki, M. A. Abbassi, A. Omri, and B. Zeghmati, "Effects of nanoparticles Brownian motion in a linearly/sinusoidally heated cavity with MHD natural convection in the presence of uniform heat generation/absorption," Powder Technology, vol. 29, pp. 69-83, 2016.

[36] M. Sheikholeslami and D. D. Ganji, "Three dimensional heat and mass transfer in a rotating system using nanofluid," Powder Technology, vol. 253, pp. 789-796, 2014.

[37] K. Singh and M. Kumar, "Effects of thermal radiation on mixed convection flow of a micro-polar fluid from an unsteady stretching surface with viscous dissipation and heat generation/absorption," International Journal of Chemical Engineering, vol. 2016, Article ID 8190234, 10 pages, 2016. 

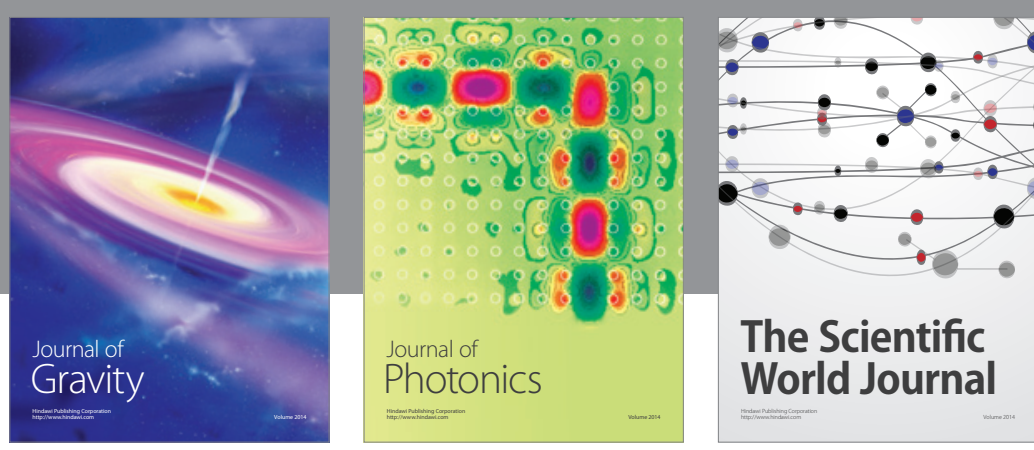

The Scientific World Journal
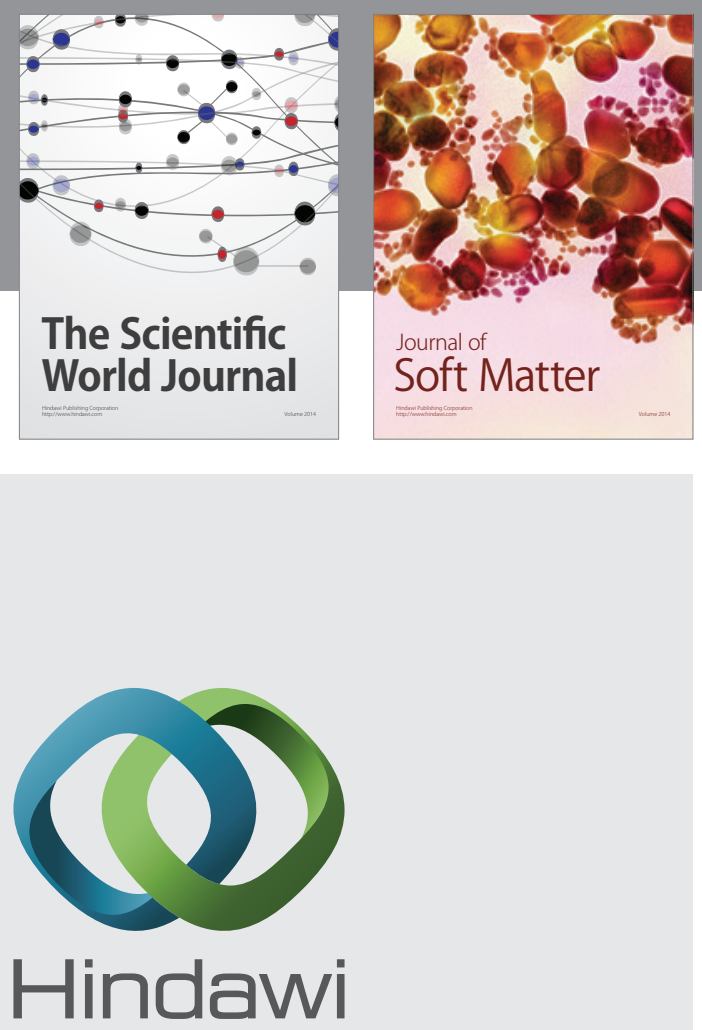

Submit your manuscripts at

http://www.hindawi.com

nternational Journal of

Statistical Mechanics
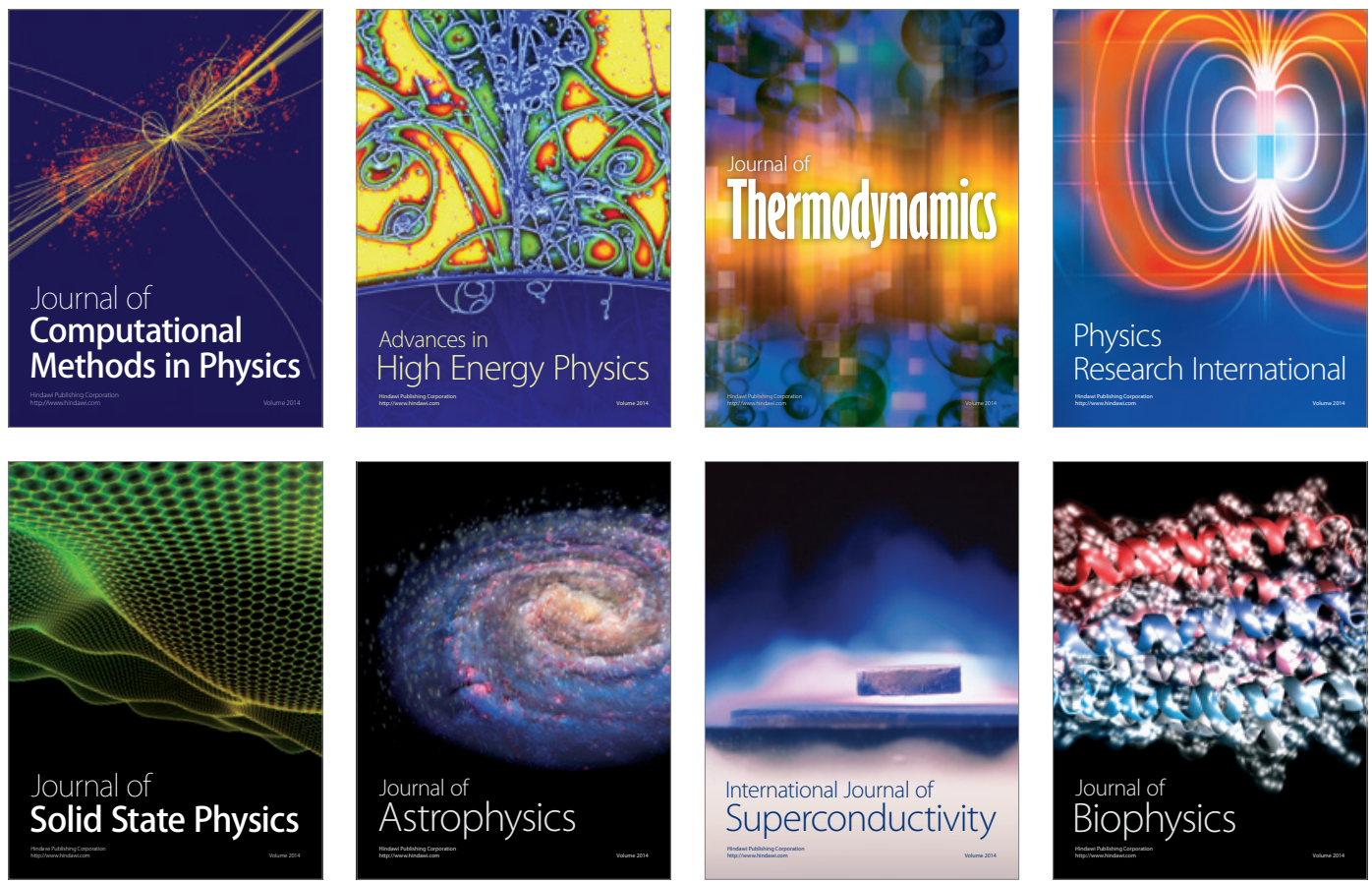
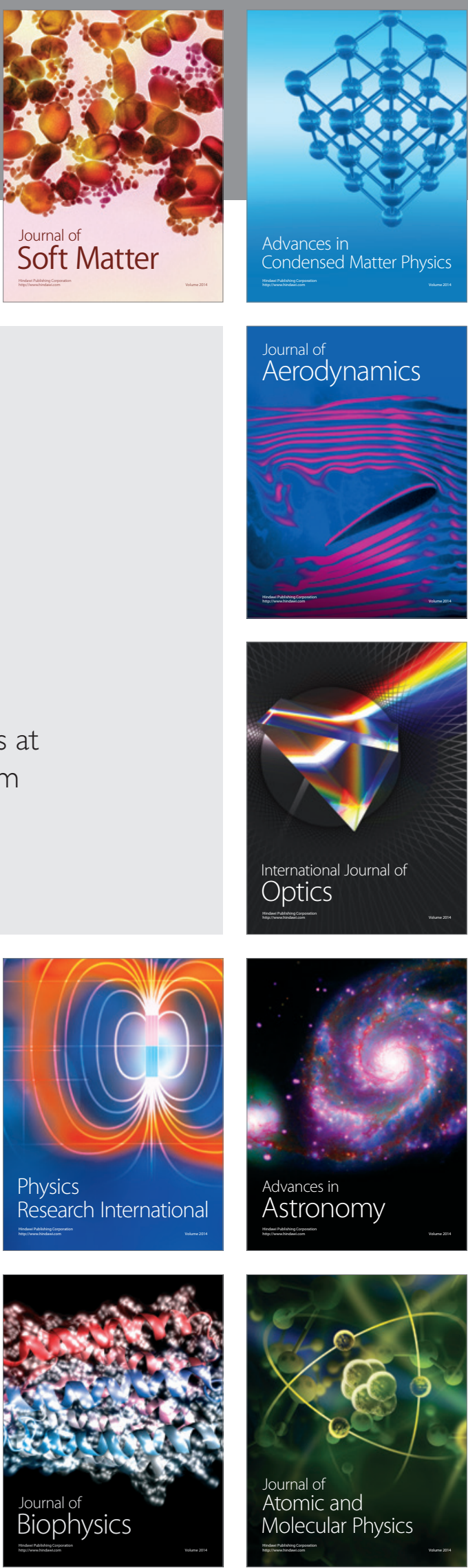